\title{
nature
}

genetics

\section{Release the flies}

I am beginning to realize that I should have prepared for a large campaign and be better organized, but who could have foreseen such a deluge. With vicarious help I have passed one acute stage only I fear to pass on to another. With what help I can muster I hope to weather the storm -Thomas Hunt Morgan, 1911

$\mathrm{T}$ wo papers in this issue by the scientists at Exelixis and the Bloomington Drosophila Stock Center (BDSC) represent what may be the largest public release of scientific material in history, in the form of some 18,000 Drosophila melanogaster stocks. Stephen Thibault and colleagues describe the creation of 29,000 P-element and piggyBac-transposable element insertion lines in an isogenic background. Approximately 16,500 of the most useful stocks will be available to researchers through the Bloomington Drosophila Stock Center and the laboratory of Spyros Artavanis-Tsakonas at Harvard University. Fifty-three percent of the 13,600 genes in the D. melanogaster genome were tagged with transposons in the collection. A second study by Annette Parks and colleagues describes the use of these lines to create 519 molecularly defined genomic deletions (deficiencies) and 45 other deficiencies generated by Kevin Cook and colleagues at Indiana University by imprecise excision of pairs of $P$ elements. All the deficiency stocks will be distributed through the BDSC.

The rumors are true: Exelixis is releasing most of their $D$. melanogaster stocks to the public. (They are keeping a few hundred stocks for further in-house analysis.) Spending money to release research material to the community seems to be outside the mission of a publicly held company. It would have been cheaper to junk the lines and take the knowledge they gained from them to quietly pursue potential drug targets. Instead, the company made the bold decision to give them to the research community. Moving a collection of that size to Indiana and Boston means shipping robots needed to transfer stocks to new media, categorizing and shipping the lines themselves and providing interim funding for upkeep of the collection during the re-establishment period. The motive behind the decision, as it turns out, is simple-the company realized the intrinsic value of the collection to researchers and decided that throwing them away would be a tremendous waste. Indeed, in the weeks preceding the publication of the papers, the company received over 150 e-mail messages per day asking for specific lines.
Exelixis was founded by academic researchers with the belief that basic model systems could be used in the discovery of new drug and agricultural targets. The release of their D. melanogaster lines, which cost millions of dollars in research-and-development funds to establish, is both evidence of the academic bent that brought Exelixis scientists to the research game and a gesture of good will by a public corporation. The fly research community will benefit immensely from their efforts.

So how do you get your mutant of interest? The Bloomington Drosophila Stock Center was offered the entire collection but selected only $\sim 2,200$ unique transposon insertion lines for distribution. Most of the insertion lines will only be available from Spyros Artavanis-Tsakonas. On page 207, he explains the terms of distribution. Establishing what amounts to a new stock center is no trivial matter, and to ensure that it can be done properly, a conservative date of 1 October 2004 is set for general distribution. It took a degree of comfort and trust to agree to send the stocks to a single investigator's laboratory, but ArtavanisTsakonas is no stranger to Exelixis, as he was one of its scientific founders and thus a logical choice to oversee the collection.

It is hard to judge the full impact of the Exelixis collection on the research community. On page 211 Hugo Bellen addresses the usefulness of the collections. The deficiency collection will probably increase the current coverage of the genome by only a few percent, but it will significantly increase the density of breakpoints within the genome and provide a substantial number of deficiencies with molecularly defined breakpoints. The two methods described for generating deficiencies will provide the tools for creating more specific aberrations than have previously been available. The Bloomington Drosophila Stock Center has taken a series of unique insertion lines into its collection that hit genes not previously hit in other transposon screens. It is not known if those insertions affect gene function in those lines.

Finally, Exelixis has also deposited information on the flanking sequence for each transposon insertion into FlyBase and has transferred EST reads from several of its randomly primed cDNA libraries to the site as well. They are also releasing several hundred transgenic lines, which the BDSC is now evaluating for inclusion in the public collection.

It would be refreshing to see corporations in other sectors show the same level of generosity that Exelixis has. 